\title{
Liderazgo y creación social: una aproximación a las principales aportaciones
}

\author{
Gisela Redondo Sama \\ Universidad de Zaragoza \\ grsama@unizar.es \\ Carmen Elboj Saso \\ Universidad de Zaragoza \\ celboj@unizar.es
}

Resumen: La conceptualización del liderazgo y la creación social son los ejes fundamentales de este artículo. Partiendo de la relevancia del liderazgo en las ciencias sociales, se realiza una revisión de literatura científica sobre liderazgo en empresas, política y movimientos sociales, y organizaciones escolares. Además, se incluyen el concepto de creación social y algunos de los elementos comunes con el liderazgo. La presentación de conceptos y los avances en estos ámbitos sugieren la necesidad de seguir investigando sobre las dinámicas de cambio implícitas en estos conceptos.

Palabras clave: liderazgo; creación social; ciencias sociales

Abstract: The conceptualization of leadership and social creation are the fundamental topics explored in this article. The relevance of leadership in the social sciences is the point of departure from which we undertake a review of the scientific literature on leadership in companies, political and social movements, and school organizations. We also include a discussion of the concept of social creation and some of the elements it shares in common with leadership. The presentation of concepts and the advances in these areas suggest the need to continue investigating the dynamics of change implicit in these concepts.

Keywords: leadership; social creation; social sciences 


\section{Introducción}

Los conceptos y desarrollos sobre liderazgo y creación social tienen recorridos históricos distintos y, a su vez, complementarios. El liderazgo, en un sentido amplio y partiendo de algunos de los desarrollos más recientes, se entiende como proceso social, económico y empresarial que implica a diversidad de perfiles, comunidades u organizaciones. El liderazgo deja de responder a unas características individuales para tender a conceptualizarse como proceso que requiere de un contexto en el que las capacidades de liderazgo se promuevan. La división entre líder y seguidor está vigente y los programas de formación para capacitar hacia el liderazgo siguen teniendo mucha presencia, aunque cada vez hay más iniciativas que potencian modelos en los que esa línea divisoria es más difusa. En definitiva, lo que se requiere para generar liderazgos es que se promueva un contexto en el que broten esas capacidades y reviertan en la mejora de la sociedad de manera sostenible. Al contrario del concepto de liderazgo, la creación social es muy reciente, aunque recupera los trabajos realizados por autores clásicos de la sociología (Merton, 1968; Weber, 1974) para incorporar avances del ámbito artístico. El objetivo de la exposición de este concepto es familiarizarse con una noción que irá adquiriendo cada vez más relevancia en las ciencias sociales.

El artículo se divide en cinco apartados. En el primero, se resume el método seguido para la selección de contribuciones. El segundo apartado se centra en una aproximación a los conceptos de liderazgo y creación social y su importancia para el análisis social. El tercer apartado profundiza en el concepto de liderazgo en las ciencias sociales, haciendo especial hincapié en el liderazgo en las empresas, los movimientos sociales y la ciencia política, y el papel de la comunidad y las organizaciones escolares. La selección de estas temáticas responde a su presencia destacada en recursos con contribuciones teóricas y empíricas en liderazgo, especialmente en Web of Science, entre otras. En cuarto lugar, se expone el concepto de creación social para vincularlo a las sinergias con los avances en la investigación sobre liderazgo. Finalmente, se exponen las conclusiones principales.

\section{Método}

Para la identificación y análisis de contribuciones relacionadas con las temáticas se ha realizado una revisión de recursos electrónicos en ciencias sociales. La selección se ha centrado en los ámbitos de la sociología, la organización, la gestión, los negocios y la educación para abarcar diferentes disciplinas de las ciencias sociales. Principalmente se ha llevado a cabo en la base de datos Web of Science - Social Sciences Citation Index (SSCI) - , aunque se han incorporado recursos elec- 
trónicos complementarios. Las contribuciones seleccionadas están mayoritariamente en inglés sin limitación temporal, ya que se pretende identificar los rasgos básicos de la evolución en la investigación sobre liderazgo. Por la naturaleza del artículo, el tipo de contribución es principalmente teórica, aunque se incorporan trabajos empíricos en la temática de liderazgo. En la búsqueda se ha tenido en cuenta cualquier ámbito geográfico y se ha procurado reflejar los avances en diferentes territorios del mundo. Las búsquedas se han realizado utilizando palabras clave, ordenando los resultados bajo tres criterios que han servido para incluir o excluir contribuciones: veces citado, relevancia y fecha.

\section{El concepto de liderazgo en las ciencias sociales}

El liderazgo es un concepto presente en las ciencias sociales que se ha vinculado en ocasiones al papel que ejercen los «líderes» en distintos ámbitos, desde la política al arte o la religión. Existe literatura científica que analiza el rol que algunos líderes han tenido a lo largo de la historia y su relación con el desarrollo del liderazgo, como por ejemplo la contribución de Carpenter (2000), que, a través del análisis de las declaraciones de presidentes de Estados Unidos desde Roosevelt hasta Clinton, identifica y profundiza en diferentes aproximaciones al liderazgo. En el ámbito de la sociología, es importante destacar la contribución de Weber en su obra publicada en 1922 Economía y sociedad, en la que establece los tipos de dominación legítima, incluyendo la dominación racional, la tradicional y la carismática. La dominación carismática está asociada a la cualidad de una personalidad, que puede ser la ejemplar del líder $(2002$ : 193) y que tiene la cualidad de extraordinaria. La existencia de aportaciones vinculadas a los líderes se acompaña de una amplia literatura sobre el desarrollo, las conceptualizaciones, las temáticas y los estilos de liderazgo que superan las aproximaciones focalizadas en el individuo.

Una de las aportaciones más citadas a nivel internacional en el ámbito de las organizaciones que clarifica la distinción entre líder y liderazgo es la realizada por David V. Day en su artículo «Leadership development: A review in context» (2000), en el que también pone de relieve la desconexión entre la práctica del liderazgo y su base científica. El autor evidencia, a través de numerosas contribuciones, la existencia de una visión tradicional de liderazgo como habilidad individual, en la que existen líderes y seguidores y de la que se desprenden programas de formación principalmente intrapersonales. Recuperando a Fiedler (1996), Day afirma sobre estos enfoques que «ignore almost 50 years of research showing leadership to be a complex interaction between the designated leader and the social and organizational environment». Partiendo de las contribuciones 
de Drath (1998), entre otros, el autor afirma que el liderazgo se entiende como proceso social que implica a la comunidad, y promueve una aproximación que conceptualiza el liderazgo como efecto, en el que es clave el papel de los miembros de una determinada comunidad. El vínculo entre el desarrollo del líder y el desarrollo del liderazgo es fundamental según el autor, que incorpora la necesidad de construir compromisos entre los miembros de la comunidad para que esos liderazgos se desarrollen y consoliden.

El papel central de la comunidad para promover el liderazgo de muy diferentes agentes está en línea con desarrollos recientes en sociología, educación, ciencia política o gestión de las organizaciones. Por tanto, se pueden identificar contribuciones que destacan la relevancia creciente del papel de las comunidades en el desarrollo y consolidación de liderazgos. Además, esa relación y desarrollos del liderazgo se investigan desde hace décadas (Burns, 1978) teniendo en cuenta, por ejemplo, la importancia que pueden tener esos desarrollos en un liderazgo de tipo transformacional, la mejora de nuestras sociedades, organizaciones, colectivos o individuos que las configuran.

\subsection{La comprensión del liderazgo en los movimientos sociales}

En el ámbito de la ciencia política y los movimientos sociales existe mucha literatura científica que vincula la relevancia que tienen los liderazgos que surgen, se desarrollan y consolidan en esos ámbitos para el cambio social. Uno de los autores de referencia a nivel internacional sobre el liderazgo en este ámbito de conocimiento es Marshal Ganz, profesor de Política Social en la Kennedy School of Government (Harvard University). En 2010 y 2011, respectivamente, Ganz publicó las contribuciones «Leading Change: Leadership, Organization and Social Movements» $y$ «Public Narrative, Collective Action, and Power», en las que analiza las formas en que el público (o la ciudadanía) puede movilizar las demandas para el cambio político a través de las «narrativas públicas». Según el autor, esto requiere que exista un proceso de adaptación, aprendizaje y conocimiento compartido que promueva la efectividad del liderazgo, de manera que se movilice a la comunidad. La red desarrollada por el autor, «The leading change network», demuestra la efectividad del liderazgo y su éxito en distintos movimientos sociales cuando se incorporan en las campañas políticas temáticas como la salud o la vivienda.

Otras contribuciones recientes vinculadas al liderazgo en los movimientos sociales son las realizadas por Kenneth, Ganz, Baggeta, Han y Lim (2010); Baggeta, Han y Andrews (2013); Harley, Metcalf y Irwin (2014) o Valls, Aubert, Puigvert y Flecha (2017). Este conjunto de autores profundiza en el liderazgo en 
los movimientos sociales, destacando diferentes aspectos. Por ejemplo, Kenneth, Ganz, Baggeta, Han y Lim están interesados en comprender la efectividad de las asociaciones cívicas en relación con el liderazgo, mientras que Harley, Metcalf e Irwin exploran los liderazgos emergentes en zonas rurales para favorecer el crecimiento sostenible. Además, Valls, Aubert, Puigvert y Flecha profundizan en el papel del liderazgo en relación con el movimiento social surgido por un caso polémico en el contexto español, como fue el de una víctima herida por una bala de goma durante una manifestación en Barcelona. Las autoras analizan el movimiento «Ojo con tu Ojo» surgido a raíz de ese caso, explorando la relevancia de las experiencias participativas previas de la víctima para comprender el éxito del movimiento. El cambio legislativo que prohíbe el uso de balas de goma por parte de la policía es el resultado que las autoras vinculan a la transferencia exitosa del conocimiento previo de la víctima en los movimientos sociales.

\subsection{La comprensión del liderazgo vinculado a la ciencia política}

El liderazgo analizado en el ámbito de la política, o liderazgo político, incluye distintos aspectos de la vida política, que son analizados desde un punto de vista más local o global. En el caso del liderazgo político local, identificamos aportaciones como la de Leach y Wilson (2002), quienes profundizan en la naturaleza cambiante del liderazgo político local en Gran Bretaña a finales del siglo xx y fundamentan la necesidad de continuar investigando en este ámbito con base en los cambios de roles y funciones de las autoridades locales, con un marcado impacto en la naturaleza del liderazgo político local. Por otro lado, desde una perspectiva global, Helms (2014) reclama la necesidad de avanzar en el conocimiento sobre el liderazgo político global, en el que distingue el liderazgo estructural (poder material de un actor) y el liderazgo conductual (acciones concretas de un posible líder), incorporando en su análisis qué actores colectivos pueden ejercer un liderazgo político global, concluyendo que son los Estados los que pueden tener una posición más favorable y sin dejar de lado el liderazgo global de redes transnacionales de la sociedad civil.

Las organizaciones políticas son también un tema de análisis en las investigaciones sobre liderazgo, con contribuciones que lo abordan de forma global, como por ejemplo Ahlquist y Levi (2011), en su contribución «Leadership: what it means, what it does, and what we want to know about it», publicada en el Annual Review of Political Science. En su análisis, parten de que los líderes son parte del conjunto de la vida política organizada y revisan las contribuciones en el ámbito poniendo en discusión hasta qué punto las aportaciones representan avances 
sobre los trabajos clásicos en liderazgo y organización en el siglo xx. Como resultado, indican la existencia de tres brechas a superar en futuras investigaciones: teorizar un rol para la coacción, modelar el surgimiento endógeno de líderes y desvelar de forma empírica el efecto de un líder individual. Este tipo de contribución se relaciona con aportaciones más generales sobre el liderazgo político.

Aunque este tipo de contribuciones se focalizan en el ámbito de la política, existen contribuciones que ponen en relación las sinergias de la política con la ciudadanía. En este sentido, Akbar y Aldrich (2015) utilizan los resultados de 450 encuestas con preguntas abiertas realizadas a residentes afectados por las inundaciones de Pakistán en 2010 para comprender las opiniones de esas personas sobre el liderazgo político. Los autores concluyen que el daño material que experimentaron las víctimas de las inundaciones puede predecir su apoyo al liderazgo político. Otra de las contribuciones que pone en relación la política con otras dimensiones en el análisis del liderazgo es la que realizan Burkett y Hayes (2018) para investigar las respuestas de administradores sobre políticas de inmigración de Donald Trump, concretamente en comunidad hispana, y la reacción de las comunidades ante esa política. En definitiva, a pesar de que este tipo de estudios empíricos son menos frecuentes en este ámbito, existen interesantes avances que complementan los desarrollos teóricos.

\subsection{La comprensión del liderazgo en las organizaciones escolares}

El papel del liderazgo en las organizaciones escolares se ha desarrollado ampliamente en la investigación educativa. La literatura científica demuestra que el liderazgo en las organizaciones escolares es uno de los factores que influyen en la mejora educativa, tanto en resultados académicos como de convivencia en los centros. Se ha investigado ampliamente sobre los distintos tipos de liderazgo (distribuido, compartido...), así como en relación con los diferentes agentes que desarrollan esos liderazgos (profesorado, alumnado, familias, comunidad...). A nivel internacional, tanto la AERA (American Educational Research Association, fundada en 1916) como la EERA (European Eduacational Research Association, fundada en 1990) tienen entre sus temáticas la investigación sobre liderazgo. Concretamente, la network 26 de la EERA está dedicada al «Educational leadership» y la Division A «Administration, Organization, and Leadership» de la AERA incluye el liderazgo educativo.

Existen diferentes aproximaciones al estudio del liderazgo en las organizaciones escolares (Marks y Printy, 2003; Pounder, Ogawa y Adams, 1995) que ponen énfasis en distintos colectivos o agentes centrales en el liderazgo de los centros 
escolares. Por ejemplo, se pueden identificar aportaciones relacionadas con el liderazgo del alumnado, de los directores de escuelas o del profesorado en general. Estas contribuciones se complementan y retroalimentan entre ellas, favoreciendo las conceptualizaciones de liderazgo en las organizaciones escolares, que trascienden lo individual para centrarse en las diferentes comunidades que las configuran.

Dempster y Lizzo (2007), en su artículo «Student leadership: necessary research», parten de la existencia del liderazgo del alumnado en los centros para argumentar la necesidad de conocer más las conceptualizaciones que los estudiantes tienen del liderazgo y en qué momentos lo consideran relevante. Los autores abordan aspectos principalmente centrados en el interés por conocer e investigar más sobre el papel del liderazgo en este colectivo, preguntándose por qué algunas de las contribuciones en este ámbito parecen carecer de cuestiones previas a plantear, como el tipo de investigación que es necesario implementar si se pretende promover el liderazgo del alumnado. En relación al liderazgo del alumnado, existen numerosas aportaciones que lo que hacen es evaluar programas de liderazgo del alumnado o identificar sus características y percepciones. Son por ejemplo los trabajos desarrollados por Chesnut y Tran-Johnson (2013), quienes evaluaron la efectividad de un programa de desarrollo de liderazgo para desarrollar habilidades de liderazgo en estudiantes de farmacia en una universidad americana, o de Tan y Adams (2018), quienes exploraron en escuelas de secundaria y possecundaria en Malasia los estilos de liderazgo de estudiantes líderes considerando las variables género, edad y etnicidad.

En el ámbito del liderazgo del profesorado y teniendo en cuenta que es en Estados Unidos donde se realizan más contribuciones a la temática, una de las aportaciones de referencia es la realizada por Muijs y Harris (2006), que profundiza en el contexto británico, explorando formas de liderazgo que influyen en los centros y en el desarrollo profesional. Los autores también definen elementos que contribuyen o dificultan que el liderazgo se desarrolle. Los datos cualitativos analizados en el artículo permiten evidenciar que el liderazgo es positivo y clave para la mejora escolar. También en el ámbito del liderazgo del profesorado se han realizado aportaciones que incluyen un tipo de liderazgo no posicional (Frost, 2014). Estos planteamientos refuerzan la existencia de liderazgos que se definen por superar las posiciones del profesorado en sus centros, promoviendo liderazgos que capacitan a diferentes perfiles de profesorado a liderar cambios y mejora escolar.

Los directores y directoras de organizaciones escolares han sido protagonistas de la línea de investigación denominada habitualmente en la literatura científica «principal leadership». Una de las contribuciones con más impacto a nivel 
internacional es la publicada por Hallinger, Bickman y Davis (1996), que relaciona el contexto escolar, el liderazgo del director y los resultados en lectura del alumnado. En su aportación, con base en una muestra de 87 escuelas de primaria de Estados Unidos, utilizan cuestionarios a directores y profesorado en relación a las calificaciones, demostrando que, aunque no se identifica un efecto directo del liderazgo instructivo de los directores y las directoras en el rendimiento del alumnado, existe un efecto indirecto en la efectividad de la escuela a través de actuaciones que dan forma al clima de aprendizaje en la escuela. De este modo, los autores confirman el papel del director en la efectividad escolar y en el rendimiento. Aportaciones más recientes de diferentes partes del mundo continúan presentando evidencias de la relación entre el liderazgo de directores y directoras y la mejora educativa en un contexto de creciente necesidad de rendición de cuentas, como la publicada por Jones et alii (2015) sobre Malasia o la analizada por Zheng, Li, Chen y Loeb (2017) en el contexto chino.

Las aportaciones sobre liderazgo en las organizaciones escolares incluyen distintas conceptualizaciones que están en línea con los desarrollos teóricos recientes en este ámbito de estudios, como el liderazgo distribuido (Gronn, 2002; Spillane, Halverson y Diamond, 2004), transformacional (Leithwood y Lanzi, 2006), compartido (Lambert, 2002) o dialógico (Padros y Flecha, 2014). Es importante tener en cuenta que de forma transversal se tiende a incorporar $y$ desarrollar investigaciones que consideran el papel que la comunidad ejerce en el liderazgo, ya sea el que se ejercita por parte de las familias, voluntariado u otros agentes que forman parte de la organización escolar y su entorno. Por tanto, se incluyen distintas formas de participación en los diferentes liderazgos en los centros. En este sentido, destaca la contribución de Warren, Hong, Rubin y Uy (2009), en la que estudiaron tres organizaciones basadas en la comunidad, concretamente en Chicago, Los Ángeles y Nueva Jersey, argumentando que el rol de esas comunidades es útil potencialmente para crear sinergias de colaboración en la organización escolar, favoreciendo el cambio.

\subsection{La comprensión del liderazgo en las empresas}

Las aportaciones teóricas y empíricas sobre el liderazgo en las empresas han estado orientado a la mejora de resultados. Goleman (2000), en una de las aportaciones más citadas a nivel internacional, publicada en Harvard Business Review, profundiza en la relación entre liderazgo y resultados. La aportación que realiza se basa en una investigación de más de 3.000 ejecutivos en la que se exploran las conductas de liderazgo que generan resultados positivos. A través de la distinción de seis tipos de liderazgo, Goleman identifica efectos diferentes en el ambiente 
laboral de una empresa, división o equipo, en vinculación también con su desempeño financiero. La conclusión principal es que los liderazgos que obtienen mejores resultados no dependen de un solo estilo y que con la práctica los líderes pueden modificar sus estilos para producir mejores resultados. En el análisis de la relación del liderazgo con los resultados, también existe literatura que profundiza en la dimensión de género, como la aportación de Haslam y Ryan (2008). Esta contribución parte de que el liderazgo en las organizaciones y el papel de la mujer se ha centrado en el análisis del denominado «techo de cristal», pero ha investigado menos el fenómeno del «acantilado de vidrio», es decir, el hecho de que las mujeres estén sobrerrepresentadas en posiciones de liderazgo precarias, asociadas a liderazgos con mayores riesgos de fracaso y crítica. Los estudios experimentales que se incluyen en esta contribución indican que la probabilidad de contratación de una mujer aumenta en comparación con un hombre con igual cualificación cuando el desempeño de la organización estaba disminuyendo en lugar de mejorar, evidenciando también asociaciones y su vinculación a las capacidades de liderazgo.

Es importante tener en cuenta que los avances en liderazgo en las empresas son incorporados como temas centrales en las escuelas de negocios y centros de investigación sobre liderazgo en las mejores universidades del mundo. En estos programas se han ido incorporando progresivamente temáticas vinculadas a la ética de los negocios publicadas en revistas como Journal of Business Ethics que, desde la diversidad de perspectivas metodológicas y disciplinarias, aporta conocimiento sobre temas éticos relacionados con los negocios y la ética empresarial, incluyendo los sistemas de producción, consumo, marketing, publicidad, contabilidad social y económica, relaciones laborales, relaciones públicas y comportamiento organizacional, que se analizan desde un punto de vista moral. Una de las contribuciones de más impacto en relación al liderazgo ético publicada en esta revista se titula «Leaders, values, and organizational climate: Examining leadership strategies for establishing an organizational climate regarding ethics» (Grojean, Resick, Dickson y Smith, 2004), y examina el papel crítico de los líderes organizacionales para establecer un clima basado en valores. A través de la discusión de siete mecanismos para transmitir la relevancia de los valores éticos, los autores argumentan la importancia de abordar la dimensión ética desde el liderazgo.

Dentro del ámbito del liderazgo en las empresas existen también estudios centrados en los negocios familiares, la evaluación de formación en liderazgo empresarial o el emprendimiento empresarial. En las empresas familiares, hay autores que han profundizado sobre los estilos de liderazgo y los valores en los negocios, 
como Venter y Farrington (2016). A través de datos obtenidos de una encuesta a más de 133 dueños de empresas familiares y 133 empresarios familiares, se identifica que, contrariamente a algunas contribuciones que centran las empresas familiares con estilos de liderazgo autocráticos, existe una alta puntuación en la valoración de estilos de liderazgo participativos y de servicio. En relación a la evaluación de formación en liderazgo empresarial, Crossan, Mazutis, Seijts y Gandz (2013) abarcan el desarrollo del carácter del liderazgo en la educación empresarial describiendo un enfoque que incluye un liderazgo a distintos niveles: individual, grupal y organizativo. Este planteamiento lo comparan con prácticas existentes para comprender las formas en que en el ámbito de la formación de negocios se permite el desarrollo de liderazgo a través de comportamientos, relaciones y estructuras. Una de las conclusiones principales que extraen es que existen distintas maneras de integrar un enfoque de liderazgo en los programas empresariales existentes, ya sea en términos de programas individualizados o en planes de estudio más generales. Por último, el emprendimiento empresarial vinculado al liderazgo también incluye desarrollos recientes, como por ejemplo el de Kesidou y Carter (2018). Los autores incluyen un análisis de seis compañías teniendo en cuenta los incidentes críticos, la dimensión temporal y el liderazgo empresarial. El análisis aborda la naturaleza evolutiva del liderazgo empresarial, demostrando que sus líderes influyen en comportamientos habilitadores, contribuyendo a la implementación de un enfoque estratégico para el espíritu empresarial.

\section{Sinergias con el liderazgo y la creación social}

El concepto de creación social es reciente y, por tanto, todavía son escasas las contribuciones vinculadas a esta aportación. A continuación, se sintetizan los principales elementos que configuran las creaciones sociales con base en la publicación de las autoras Aiello y Joanpere titulada «Social Creation. A New Concept for Social Sciences and Humanities» (2014), que diferencian entre las nociones de diseminación, transferencia, impacto y creación social.

El concepto de creación social implica que como resultado de la investigación social «surgen nuevas realidades sociales exitosas que mejoran la sociedad en formas que hasta aquel momento no habían existido» (p. 298). Partiendo de las aportaciones de clásicos de la sociología, las autoras relacionan los avances de la investigación europea con las creaciones sociales. Concretamente, desarrollan algunas de las contribuciones resultantes del proyecto IMPACT-EV. Evaluating the impact and outcomes of European SSH research del 7. Programa Marco (IMPACT-EV Consortium, 2014-2018), que con la dirección de Ramón Flecha ha tenido como objetivo desarrollar indicadores y criterios que están sirviendo para 
evaluar no solo el impacto científico de la investigación en las ciencias sociales y las humanidades, sino especialmente sus impactos sociales y políticos. Además, incluyen como aspecto central del artículo el concepto de creación social y la evaluación de las ciencias sociales y humanidades, con base en la aportación de Flecha (2014), reproducida a continuación:

Las ciencias sociales necesitan dar un salto cualitativo sobre lo que han sido hasta ahora no solo para contribuir claramente a la mejora de la sociedad sino también para superar el creciente cuestionamiento que la propia sociedad está haciendo de su validez, incluso de la necesidad de su existencia. Algunos ámbitos de humanidades tienen el concepto de creación artística que deja claro la aportación que, por ejemplo, personas como Picasso han hecho a la humanidad, aunque nunca hayan hecho ningún artículo o libro. En algunas ciencias también está claro que el valor de un artículo no está en su redactado sino en el descubrimiento que explica, por ejemplo, el artículo que hace un año comunicó la obtención de células madre embrionarias a partir de una célula adulta en humanos.

Las ciencias sociales requieren el concepto de creación social que deje muy claras las aportaciones que están haciendo a la mejora de la realidad social. Por ejemplo, un mismo autor ha podido hacer un artículo sobre el modelo cooperativo de Mondragón y otro sobre las Tertulias Literarias Dialógicas. En la actualidad, ambos tienen el mismo mérito si son del mismo cuartil de JCR y con el mismo impacto. Sin embargo, la aportación no es la misma si el primer artículo explica una realidad social que su autor no ha contribuido a crear ni a desarrollar y, por el contrario, el segundo explica una realidad social que él ha creado. Incluso las formas actuales de evaluación no dan ningún mérito a quien sí creó el modelo Cooperativo Mondragón, puesto que no publicó ningún artículo sobre este tema (Flecha, 2014).

Se pueden identificar distintas sinergias entre el liderazgo y la creación social. En primer lugar, hay un elemento transversal en muchas de las contribuciones sobre liderazgo que abordan la parte moral o ética de los avances en investigación sobre liderazgo, como los que se han identificado sobre la ética empresarial o la necesidad de mejora social. En este sentido, se identifica también que la creación social lleva implícita una coherencia de los científicos y científicas sociales, y por tanto, tiene relación también con la dimensión ética de nuestras actividades profesionales. En segundo lugar, se identifica una necesidad de orientar los trabajos hacia la mejora social, tanto en distintos tipos de liderazgo como en la definición del concepto de creación social. En consecuencia, hay elementos que apuntan a que esas mejoras van a ser cada vez más importantes en los desarrollos tanto del liderazgo como de la creación social. Finalmente, el reconocimiento social tanto 
de las personas que lideran como de los creadores sociales es una temática que se identifica en ambas líneas de investigación. En ambos casos, se buscar una imagen de liderazgo o creación social que refuerce positivamente su sostenibilidad y continuo crecimiento.

\section{Limitaciones}

La aproximación teórica a los conceptos que se desarrollan en este artículo presenta algunas limitaciones. En primer lugar, es importante destacar que el concepto de creación social es muy reciente y, por tanto, la aproximación a sus distintas dimensiones es todavía limitada. A medida que se avance en este concepto y sus implicaciones prácticas, se podrán establecer más vinculaciones con el análisis del liderazgo en ese contexto. Este avance se verá enriquecido por los estudios empíricos que con base en el concepto de creación social se desarrollen, así como de los casos de éxito. En segundo lugar, las aportaciones en forma de libro o capítulo de libro están menos presentes en este artículo. En la medida en que se digitalicen en acceso abierto este tipo de contribuciones, podrán ampliarse los desarrollos en los ámbitos del liderazgo y la creación social. Las iniciativas de open books que se están impulsando por parte de algunas de las editoriales más importantes del mundo facilitarán esta labor. En tercer lugar, el hecho de que la mayoría de publicaciones se realice en inglés supone que pueden existir aportaciones en otros idiomas que no se incorporen en esta aproximación a los conceptos y sus implicaciones. Finalmente, en la temática de liderazgo, se ha acotado a términos y ámbitos de manera que existen estilos de liderazgo o temas que pueden explorarse en el futuro.

\section{Conclusiones}

Los conceptos de liderazgo y creación social contribuyen a la comprensión de la realidad social y a algunas de sus transformaciones más recientes. A lo largo del artículo, se ha podido identificar la evolución del liderazgo en algunos de los principales ámbitos de conocimiento y temas de interés en ciencias sociales, destacando algunos de sus principales teóricos y teóricas a nivel internacional. La comprensión del liderazgo se ha acompañado de la definición y desarrollo del concepto de creación social, a través de la principal contribución que acota el uso del término estableciendo una muy interesante comparativa entre ciencias sociales y ciencias naturales. Si consideramos los avances en liderazgo vinculados a la temática de la creación social, pueden surgir nuevos temas sobre los que investigar. Estos avances hay que situarlos en un contexto de creciente relevancia de 
la investigación con impacto social. En este sentido, se han incorporado en este artículo algunos de los trabajos que presentan evidencias de cómo la investigación en liderazgo revierte en la mejora de resultados en las organizaciones, entre otros. También en la temática de la creación social existe una vinculación con ese impacto de la investigación, ya que la identificación de esas creaciones sociales está relacionada con el aumento del bienestar de determinados colectivos.

Para terminar, destacar que ambos conceptos serán cruciales para comprender no solo las dinámicas de cambio y transformación sino también para incorporar las voces de quienes son los y las protagonistas de esos cambios. De alguna manera, también se trata de que los avances en liderazgo y creación social puedan reflejar y dar visibilidad a la diversidad de agentes que los configuran, desde un profesional que lidera la inclusión cultural en una organización empresarial hasta el liderazgo de una voluntaria de una organización educativa que impulsa formación para las familias o el papel de un creador social que genera mejoras sociales tras la creación de una cooperativa. El avance de las ciencias sociales en liderazgo y creación social continúan abriendo nuevos horizontes de ampliación de conocimiento que revierta en la mejora social. 


\section{Bibliografía}

Aiello, E. y Joanpere, M. (2014). «Social Creation. A New Concept for Social Sciences and Humanities». Multidisciplinary Journal of Social Sciences, 3(3), 297-313. DOI: 10.4471/rimcis.2014.41.

Ahlquist, J. S. y Levi, M. (2011). «Leadership: What It Means, What It Does, and What We Want to Know About It». Annual Review of Political Science, 14, 1-24. DOI: 10.1146/annurev-polisci-042409-152654.

Akbar, M. S.y Aldrich, D. P. (2015). «Flood Damage and Victims' Perceptions About Political Leadership». Risk Hazards E Crisis in Public Policy, 6(4), 329-343. DOI: 10.1002/rhc3.12089.

Baggeta, M.; Han, H., y Andrews, K. T. (2013). «Leading Associations: How Individual Characteristics and Team Dynamics Generate Committed Leaders». American Sociological Review, 78(4), 544-573. DOI: $10.1177 / 0003122413489877$.

Burkett, J.y Hayes, S. (2018). «Campus Administrators' Responses to Donald Trump's Immigration Policy: Leadership during Times of Uncertainty». International Journal of Educational Leadership and Management, 6(2), 98125. DOI: 10.17583/ijelm.2018.3602.

Burns, J. M. (1978). Leadership. New York: Harper \& Row.

Carpenter, D. M. (2000). «Presidents of the United States on Leadership». Leadership, 3(3), 251-280. DOI: 10.1177/1742715007079307.

Chesnut, R. y Tran-Johnson (2013). «Impact of a Student Leadership Development Program». American Journal of Pharmaceutical Education, 77(10), 225. DOI: 10.5688/ajpe7710225.

Crossan, M.; Mazutis, D.; Seijts, G. y Gandz (2013). «Developing Leadership Character in Business Programs». Academy of Management Learning E Education, 12(2), 285-305. DOI: 10.5465/ amle.2011.0024A.

DAY, D. (2000). «Leadership development: A review in context», Leadership Quarterly, 11(4), 581-613. DOI: 10.1016/S1048-9843(00)00061-8.

Dempster, N. y Lizzio, A. (2007). «Student leadership: necessary research». Australian Journal of Education, 51(3), 276-285. DOI: 10.1177/000494410705100305.

Drath, W. H. (1998). «Approaching the future of leadership development». En McCauley, C. D.; Moxley, R. S. y Van Velsor, E. (eds.), The Center for Creative Leadership handbook of leadership development (pp. 403-432). San Francisco: Jossey-Bass. 
Fiedler, F. E. (1996). «Research on leadership selection and training: One view of the future». Administrative Science Quarterly, 41, 241-250.

Furió, V. (2012). Arte y reputación: Estudios sobre el reconocimiento artístico. Bellaterra: Universitat Autònoma de Barcelona.

IMPACT-EV Consortium (2014-2018). IMPACT-EV: Evaluating the impact and outcomes of European SSH research ( $7^{\text {th }}$ Framework Program, Socio-economic Sciences and Humanities. Project No 613202). Brussels: Directorate-General for Research \& Innovation, European Commission.

Flecha, R. (2014). [Debate general]. «El concepto de Creación Social y evaluación Ciencias Sociales y Humanidades». [Message 1]. [Online forum comment]. Consultado en: <http://amieedu.org/debate/index.php?action=pro file; are $=$ showposts;sa $=$ topics; $\mathrm{u}=11>$.

Frost, D. (2014). Transforming education through teacher leadership. Cambridge: Leadership for Learning.

Ganz, M. (2010). «Leading Change: Leadership, Organization and Social Movements». En Nohria, N. y Krurana, R. (eds.), Handbook of Leadership Theory and Practice. Danvers: Harvard Business School Press.

- (2011). «Public Narrative, Collective Action, and Power». En Odugbemi, S. y Lee, T. (eds.). Accountability through Public Opinion: From Inertia to Public Action (pp. 273-289). Washington, DC: World Bank Group Publications.

Goleman, D. (2000). «Leadership that gets results». Harvard Business Review, $78(2), 78$

Grojean, M.; Resick, C.; Dickson, M. y Smith, D. (2004). «Leaders, values, and organizational climate: Examining leadership strategies for establishing an organizational climate regarding ethics». Journal of Business Ethics, 55(3), 223-241. DOI: 10.1007/s10551-004-1275-5.

Gronn, P. (2002). «Distributed leadership as a unit of analysis». Leadership Quarterly, 13(4), 423-451. DOI: 10.1016/S1048-9843(02)00120-0.

Hallinger, P.; Bickman, L. y Davis, K. (1996). «School context, principal leadership, and student reading achievement». Elementary school journal, 96(5), 527-549. DOI: 10.1086/461843.

Harley, C.; Metcalf, L. e Irwin, J. (2014). «An Exploratory Study in Community Perspectives of Sustainability Leadership in the MurrayDarling Basin». Journal of Business Ethics, 124(3), 413-433. DOI: 10.1007/s10551013-1877-X.

Haslam, S. A. y Ryan, M. K. (2008). «The road to the glass cliff: Differences in the perceived suitability of men and women for leadership positions in succeeding and failing organizations». Leadership Quarterly, 19(5), 530-546. DOI: 10.1016/j.leaqua.2008.07.011. 
Helms, L. (2014). "Global political leadership in the twenty-first century: problems and prospects». Contemporary Politics, 20(3), 261-277. DOI: 10.1080/13569775.2014.911499.

Jones, M.; Adams, D;; Joo, M. T. H.; Muniandy, V.: Perera, C. J.y Harris, A. (2015). «Contemporary challenges and changes: principals' leadership practices in Malaysia». Asia Pacific Journal of Education, 35(3), 353-365. DOI: 10.1080/02188791.2015.1056591.

Kenneth, A.; Ganz, M.; Baggetta, M.; Han, H. y Lim, C. (2010). «Leadership, Membership, and Voice: Civic Associations That Work». American Journal of Sociology, 115(4), 1.191-1.242. DOI: 10.1086/649060.

Kesidou, E. y Carter, S. (2018). «Entrepreneurial Leadership: An Exploratory Study of Attitudinal and Behavioral Patterns over the Business Life-Cycle». International Review of Entrepreneurship, 16(1), 63-88.

Lambert, L. (2002). "A framework for shared leadership». Educational Leadership, 59(8), 37-40.

Leach, S. y Wilson, D. (2002). «Rethinking local political leadership». Public Administration, 80(4), 665-689. DOI: 10.1111/1467-9299.00323.

Leitwhood, K. y Jantzi, D. (2006). «Transformational school leadership for large-scale reform: Effects on students, teachers, and their classroom practices». School effectiveness and school improvement, 17(2), 201-227. DOI: 10.1080/09243450600565829.

Marks, H. M. y Printy, S. M. (2003). «Principal leadership and school performance: An integration of transformational and instructional leadership». Educational Administration Quarterly, 39(3), 370397. DOIः 10.1177/0013161X03253412.

Merton, K. R. (1968). «The Matthew Effect in Science». Science, 159(381), 56-63.

Muijs, D. y Harris, A. (2006). «Teacher led school improvement: Teacher leadership in the UK». Teaching and Teacher Education, 22(8), 961-972. DOI: $10.1016 /$ j.tate.2006.04.010.

Padrós, M. y Flecha, R. (2014). «Towards a Conceptualization of Dialogic Leadership». International Journal of Educational Leadership and Management, 2(2), 207-226. DOI: 10.4471/ijelm.2014.17.

Pounder, D. G.; Ogawa, R. T. y Adams, E. A. (1995). «Leadership as an organization-wide phenomena - Its impact on school performance». Educational Administration Quarterly, 31(4), 564-588. DOI: 10.1177/0013161X9503100404. 
Spillane, J. P.; Halverson, R. y Diamond, J. B. (2004)+ «Towards a theory of leadership practice: a distributed perspective». Journal of Curriculum Studies, 36(1), 3-34. DOI: 10.1080/0022027032000106726.

TAN, M. H. J. y Adams, D. (2018). «Malasyan student leaders' perception of their leadership styles». International Journal of Innovation and Learning, 23(3), 368-382. DOI: 10.1504/IJIL.2018.10010776.

Valls, R.; Aubert, A.; Puignert, L. y Flecha, A. (2017). «Leadership in Social Movements: The Case of Ojo con tu Ojo». International Journal of Educational Leadership and Management, 5(2), 148-177. DOI: 10.17583/ ijelm.2017.2751.

Venter, E. y Farrington, S. M. (2016). «Investigating valueladen leadership styles among family business owners». South African Journal of Business Management, 47(4), 35-46.

Warren, M. R.; Hong, S.; Rubin, C. L. y Uy, P. S. (2009). «Beyond the Bake Sale: A Community-Based Relational Approach to Parent Engagement in Schools». Teachers College Record, 111(9), 2.209-2.254.

Weber, M. (2002, p.o. 1922). Economía y sociedad. Madrid: Fondo de Cultura Económica de España.

- (1974). Sobre la teoría de las ciencias sociales (2. a ed.). Barcelona: Ediciones Península.

- (2012, p.o, 1919). La ciencia como profesión, la política como profesión. Madrid: Alianza.

Zheng, Q*; Li, L. Y*; Chen, H. J. y Loeb, S. (2017). «What aspects of Principal Leadership are most highly correlated with school outcomes in China?» Educational Admonistration Quarterly, 53(3), 409-447. DOI: 10.1177/0013161X17706152. 